\title{
Conference Calendar
}

\section{Journal of Vascular \\ Research}

15-17 April 2007

7th Asian Congress for Microcirculation ACM'07

incorporating the 6th Chinese National Congress for Microcirculation

Hang Zhou, China

E-Mail: acm07@mail.bme.zju.edu.cn

http://acm07.zju.edu.cn/

13-17 June 2007

MEET 2007

Multidisciplinary European Endovascular Therapy

Building Endovascular Synergies

Cannes, French Riviera

Organizers: Max Amor, Patrice Bergeron, Luigi Inglese,

Thomas Ischinger, Klaus Mathias, Dieter Raithel

E-Mail: jgay@meetcongress.com

http://www.meetcongress.com
4-8 August 2007

Gap Junction Conference

Kopenhagen, Denmark

Info: Niels-Henrik Holstein-Rathlou

E-Mail: niels@mfi.ku.dk

http://www.gapjunction2007.dk

15-19 August 2007

8th World Congress for Microcirculation

Milwaukee, Wisc., USA

Info:microcirc@mcw.edu

http://www.microcirccongress.org/

12-14 October 2007

12th Annual Meeting of the European Council for Cardiovascular Research (ECCR)

La Colle sur Loup, Nice, France

Info: eccr@hamptonmedical.com

www.eccr.org 